\title{
Successful Treatment of Ulcerative- Type Pyoderma Gangrenosum with a Combination Therapy of Oral Prednisolone, Vacuum-Assisted Closure, and Skin Grafting
}

\author{
Hiroyuki Goto ${ }^{a}$ Yoshimoto Okada $^{b}$ Seimi Watanabe ${ }^{a} K_{\text {Kanako Danno }}^{b}$ \\ Shinya Yamamoto ${ }^{a}$ Ryohei Ishiura $^{b}$ Yuichi Nakayama ${ }^{a} \quad K$ Kohei Mitsui ${ }^{b}$ \\ Ayaka Ueki $^{a}$ Yasuo Nakai ${ }^{a}$ Mitsunaga Narushima ${ }^{b}$ Keiichi Yamanaka ${ }^{a}$ \\ aDepartment of Dermatology, Mie University Graduate School of medicine, Mie, Japan; \\ bPlastic and Reconstructive Surgery, Mie University Graduate School of medicine, Mie, \\ Japan
}

Keywords

Pyoderma gangrenosum $\cdot$ Corticosteroid $\cdot$ Vacuum-assisted closure $\cdot$ Skin grafting

\begin{abstract}
Pyoderma gangrenosum $(P G)$ is a relatively rare inflammatory skin disease that progressively causes necrotic ulceration mainly on the lower extremities and trunk. Systemic corticosteroid is considered a first-line treatment for PG as it facilitates wound healing; however, several cases where tumor necrosis factor- $\alpha$ inhibitors, adalimumab and infliximab, were administered showed good response. For intractable PG with a large ulcer or problematic epithelization, chemical or mechanical debridement of necrotic tissue in combination with skin grafting may be necessary to promote wound healing. Our report presents a case of intractable ulcerative $P G$ requiring oral prednisolone and skin grafting. Although mechanical debridement was performed, granulation was poor; therefore, after the PG activity became quiescent, we utilized a vacuum-assisted closure (VAC) system to promote granulation and adaptation of the grafted skin. Although more cases are required for a definitive conclusion, the VAC system may be a choice for PG with large ulcers.
\end{abstract}


Goto et al.: VAC Therapy for Pyoderma Gangrenosum

\section{Introduction}

Pyoderma gangrenosum (PG) is a relatively rare inflammatory skin disease that progressively causes necrotic deep painful ulceration mainly on the lower extremities and trunk [1]. PG affects approximately 3-10 people per million annually, mainly young and middle-aged adults [1-3]. Although the pathophysiology of PG is incompletely understood, activated neutrophils and inflammatory cytokines have been shown to be involved, accompanied with a genetic background [4,5]. Approximately half of PG cases develop on a background of systemic diseases, such as rheumatoid arthritis, inflammatory bowel diseases, and hematological diseases [1-3]. A skin biopsy confirming the presence of neutrophilic infiltrate and absence of vasculitis are frequently required for a definitive diagnosis of $P G$ $[1,5-7]$.

Systemic corticosteroids or cyclosporine are considered first-line treatments for PG [1, 5] as they facilitate wound healing in PG; however, several cases showed unresponsiveness, and tumor necrosis factor- $\alpha$ inhibitors, adalimumab and infliximab, have been introduced and have shown good response in the usual cases. Large ulcers take a long time to heal; therefore, surgical treatment is occasionally considered after PG activity is clinically quiescent, although surgery itself may worsen PG $[1,8]$. The removal of necrotic tissue might be important for accelerating granulation and epithelization, and skin grafting would be preferable because epithelization from the surrounding tissue may take a long time.

Here, we present a case of ulcerative PG. Although chemical and mechanical debridement of necrotic tissue was performed, granulation was poor. Therefore, after the PG activity became quiescent, we utilized a vacuum-assisted closure (VAC) system to promote granulation, and skin grafting was then performed. Although a larger number of cases are required to reach a definite conclusion, our report suggests that the VAC system could be used for large size of ulcerative PG.

\section{Case Report/Case Presentation}

A 68-year-old woman was referred to our department because of an ulcer of the right lower extremity that was first noticed 6 months previously without any trigger. She had a history of multiple sclerosis, neuromyelitis optica, and myasthenia gravis and was taking prednisolone $12 \mathrm{mg}$ ( $0.26 \mathrm{mg}$ per $\mathrm{kg}$ ) and tacrolimus hydrate $6 \mathrm{mg}$ daily. On physical examination, there was a large ulcer in the right lower extremity. Skin infection was excluded based on the result of bacterial culture. The C-reactive protein (CRP) level was slightly elevated $(0.18 \mathrm{mg} / \mathrm{dL})$. After confirming the diagnosis by skin biopsy, we increased the prednisolone dosage to $22 \mathrm{mg}$ ( $0.47 \mathrm{mg}$ per $\mathrm{kg}$ ) and maintained the tacrolimus hydrate at $6 \mathrm{mg}$ daily (shown in Fig. 1a, week 0). The CRP level notably decreased, and new ulcer formation ceased. Thereafter, the prednisolone and tacrolimus hydrate were gradually decreased, but a reduction in the ulcer area was not observed. By week 6, the CRP level was $0.10 \mathrm{mg} / \mathrm{dL}$, and the PG activity had decreased with prednisolone $12 \mathrm{mg}(0.26 \mathrm{mg}$ per $\mathrm{kg})$ and tacrolimus hydrate $2 \mathrm{mg}$ daily. Mechanical debridement for the thick necrotic tissue was performed (shown in Fig. 1b), followed by the use of the VAC ULTA ${ }^{\circledR}(3 \mathrm{M} / \mathrm{KCI}$, Tokyo, Japan) system for 1 week (shown in Fig. 1c). In week 8, along with regional lymphatic vessel ligation for lymphatic leakage, the full layer skin grafting from the thigh was performed in combination with the VAC system (shown in Fig. 1d). By week 16, the patient was still receiving prednisolone $12 \mathrm{mg}(0.26 \mathrm{mg}$ per $\mathrm{kg}$ ) and tacrolimus hydrate $2 \mathrm{mg}$ daily, and the skin ulcer was well controlled without relapse (shown in Fig. 1e). The epithelization at the site of skin sampling was completed, and CRP level was within the normal range.

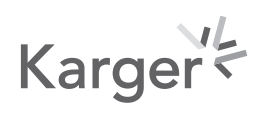



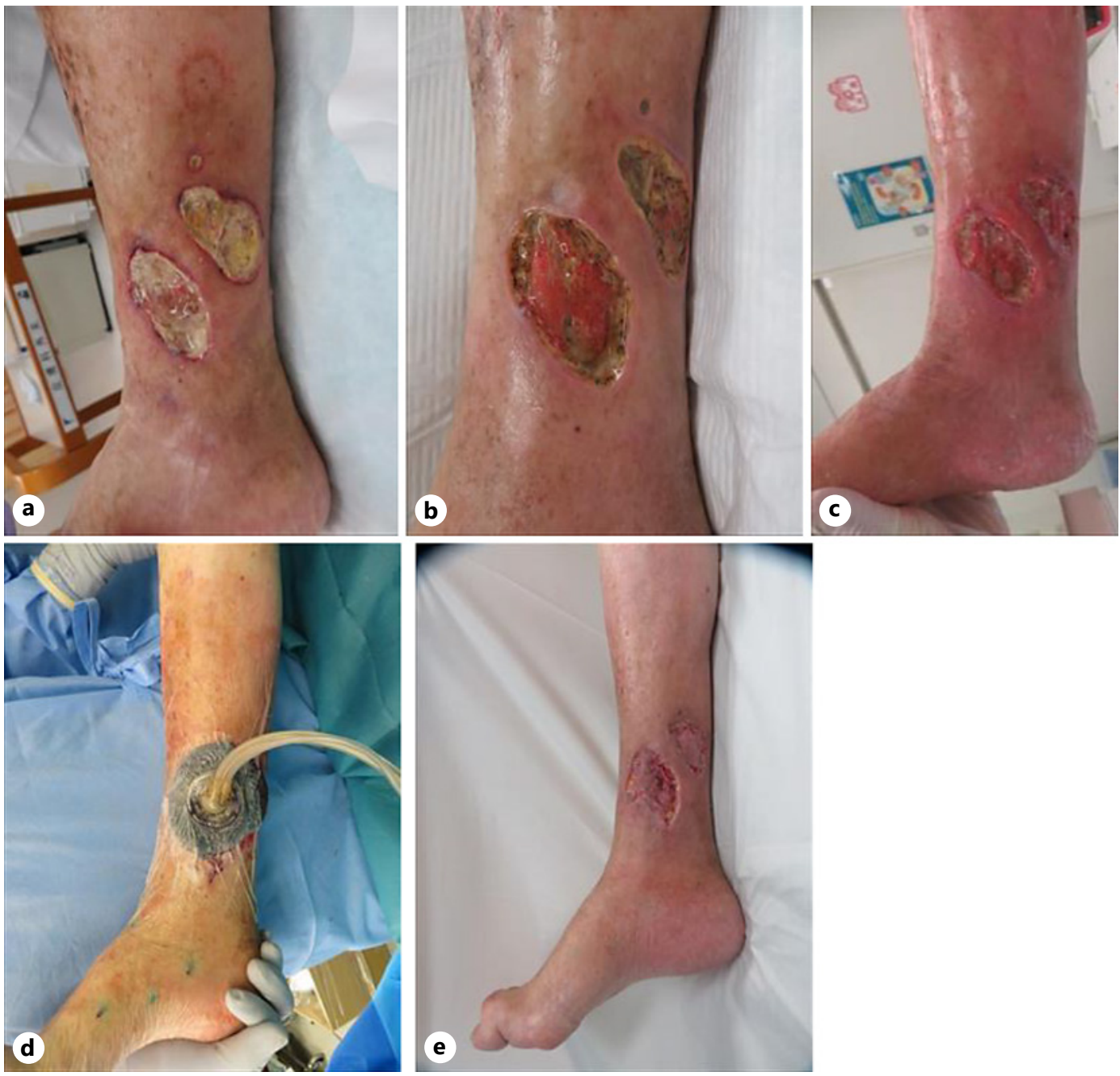

Fig. 1. a On physical examination in week 0 , there was a large ulcer of the right lower extremity. b In week 6, mechanical debridement for the thick necrotic tissue was performed. c After a 1-week use of the VAC ULTA $^{\circledR}$ system, granulation was promoted. d In week 8, skin grafting combined with the VAC system was performed. e By week 16, the skin ulcer was well controlled without relapse.

\section{Discussion/Conclusion}

Moderate-dose systemic corticosteroid has long been used for the treatment of PG. However, because of several adverse effects [1,9], adalimumab was adopted as a treatment owing to its effectiveness and safety compared to oral corticosteroid with regard to the healing speed of intractable ulcer $[10,11]$.

In the current case, adalimumab was contraindicated because of the history of multiple sclerosis. Although the activity of PG was controlled with oral steroids and CRP decreased to the normal range, the lack of granulation made it necessary to consider the following. In PG, it is well known that minor trauma can lead to new lesions, a phenomenon known as pathergy. Therefore, there is a risk of surgical procedures inducing new ulcer lesions. This informed the use of the VAC system in this case, with sufficient immunosuppression and attention to disease activity markers such as CRP. In a normal VAC system, the environment is closed and the risk of inducing infection is high, although local infection was excluded. Therefore, we 
used a system with a microenvironment, negative pressure closure therapy, and a cleaning function with the cyclic automatic injection of cleaning solution. Although this case is a specific situation in which adalimumab was contraindicated because of the past history, and longterm follow-up is required to monitor for recurrence, the current VAC system may be useful for treating intractable PG with large ulcers, occasionally in combination with skin grafting.

In conclusion, in case of PG with large ulcers, the removal of necrotic tissue and the VAC system may be important for accelerating granulation and epithelization. Moreover, skin grafting is preferable because epithelization from the surroundings may take a long time.

\section{Statement of Ethics}

Written informed consent was obtained from the patient for the publication of this case report and any accompanying images. The study is exempt from ethics committee approval because it is a single case report.

\section{Conflict of Interest Statement}

M. Narushima received a chair fee of a local meeting from 3M/KCI. Other authors declare no conflicts of interest.

\section{Funding Sources}

This research received no external funding.

\section{Author Contributions}

H.G. contributed to conceptualization, project administration, and writing - original draft; Y.O. contributed to supervision; S.W. contributed to supervision; K.D. contributed to supervision; S.Y. contributed to supervision; R.I. contributed to supervision; Y.N. contributed to supervision; K.M. contributed to supervision; A.U. contributed to supervision; Y.N. contributed to supervision; M.N. contributed to conceptualization, project administration, and writing - review and editing; K.Y. contributed to conceptualization, project administration, and writing - review and editing.

\section{Data Availability Statement}

The patient data are not publicly available on legal or ethical grounds.

\section{References}

1 Ahronowitz I, Harp J, Shinkai K. Etiology and management of pyoderma gangrenosum: a comprehensive review. Am J Clin Dermatol. 2012;13:191-211.

2 Ruocco E, Sangiuliano S, Gravina AG, Miranda A, Nicoletti G. Pyoderma gangrenosum: an updated review. J Eur Acad Dermatol Venereol. 2009;23:1008-17.

3 Yamamoto T. Epidemiology of pyoderma gangrenosum in Japanese patients by questionnaire survey. J Dermatol. 2019;46:e145-6. 
4 Braun-Falco M, Kovnerystyy O, Lohse P, Ruzicka T. Pyoderma gangrenosum, acne, and suppurative hidradenitis (PASH): a new autoinflammatory syndrome distinct from PAPA syndrome. J Am Acad Dermatol. 2012; 66:409-15.

5 Alavi A, French LE, Davis MD, Brassard A, Kirsner RS. Pyoderma gangrenosum: an update on pathophysiology, diagnosis and treatment. Am J Clin Dermatol. 2017;18:355-72.

6 Gameiro A, Pereira N, Cardoso JC, Gonçalo M. Pyoderma gangrenosum: challenges and solutions. Clin Cosmet Investig Dermatol. 2015;8:285-93.

7 Su WP, Davis MD, Weenig RH, Powell FC, Perry HO. Pyoderma gangrenosum: clinicopathologic correlation and proposed diagnostic criteria. Int J Dermatol. 2004;43:790-800.

8 Nakayama Y, Akeda T, Iida S, Habe K, Yokota N, Matsushima Y, et al. Whether to maintain or strengthen the treatment for pyoderma gangrenosum ulcerative type may depend on the response after two to four-week treatment intervention: the outcome of three cases with details clinical course. Clin Case Rep. 2021 Aug 21; 9(8):e04690. in press

9 Wang AS, Armstrong EJ, Armstrong AW. Corticosteroids and wound healing: clinical considerations in the perioperative period. Am J Surg. 2013;206:410-7.

10 Yamasaki K, Yamanaka K, Zhao Y, Iwano S, Takei K, Suzuki K, et al. Adalimumab in Japanese patients with active ulcers of pyoderma gangrenosum: twenty-six-week phase 3 open-label study. J Dermatol. 2020;47: 1383-90.

11 Ormerod AD, Thomas KS, Craig FE, Mitchell E, Greenlaw N, Norrie J, et al. Comparison of the two most commonly used treatments for pyoderma gangrenosum: results of the STOP GAP randomised controlled trial. BMJ. 2015;350:h2958.

\section{Karger ${ }^{\prime}$}

